PROCEEDINGS OF THE

AMERICAN MATHEMATICAL SOCIETY

Volume 138, Number 12, December 2010, Pages 4231-4242

S 0002-9939(2010)10465-3

Article electronically published on July 9, 2010

\title{
DIVISIBILITY THEORY OF SEMI-HEREDITARY RINGS
}

\author{
P. N. ÁNH AND M. SIDDOWAY
}

(Communicated by Birge Huisgen-Zimmermann)

\begin{abstract}
The semigroup of finitely generated ideals partially ordered by inverse inclusion, i.e., the divisibility theory of semi-hereditary rings, is precisely described by semi-hereditary Bezout semigroups. A Bezout semigroup is a commutative monoid $S$ with 0 such that the divisibility relation $a \mid b \Longleftrightarrow b \in a S$ is a partial order inducing a distributive lattice on $S$ with multiplication distributive on both meets and joins, and for any $a, b, d=a \wedge b \in S, a=d a_{1}$ there is $b_{1} \in S$ with $a_{1} \wedge b_{1}=1, b=d b_{1} . S$ is semi-hereditary if for each $a \in S$ there is $e^{2}=e \in S$ with $e S=a^{\perp}=\{x \in S \mid a x=0\}$. The dictionary is therefore complete: abelian lattice-ordered groups and semi-hereditary Bezout semigroups describe divisibility of Prüfer (i.e., semi-hereditary) domains and semi-hereditary rings, respectively. The construction of a semi-hereditary Bezout ring with a pre-described semi-hereditary Bezout semigroup is inspired by Stone's representation of Boolean algebras as rings of continuous functions and by Gelfand's and Naimark's analogous representation of commutative $C^{*}$ algebras.
\end{abstract}

\section{INTRODUCTION}

In considering the structure of rings, classical number theory suggests a careful study of the semigroup of divisibility, defined as the semigroup of principal (or more generally finitely generated) ideals partially ordered by reverse inclusion. We refer to this special semigroup of ideals in a ring $R$ as the divisibility theory of $R$, or more informally as its divisibility. This approach led to a general valuation theory developed by Krull, establishing a dictionary between valued fields and totally ordered abelian groups. Later, valuation theory is extended to Bezout domains by showing that the groups of divisibility (consisting of fractional ideals) of such domains are precisely lattice-ordered abelian groups. However, the task of describing a divisibility theory for Bezout rings (rings whose finitely generated ideals are principal) with zero-divisors appears to be far more difficult and is still open, although it was quite simple to settle the problem for valuation rings (rings, possibly with zero-divisors, in which any two ideals are comparable).

Received by the editors December 11, 2009 and, in revised form, February 25, 2010.

2000 Mathematics Subject Classification. Primary 13A05, 13D05, 13F05; Secondary 06F05.

Key words and phrases. Bezout rings, Stone space, semi-hereditary, rings of continuous functions.

The first author was partially supported by the Hungarian National Foundation for Scientific Research grants no. K61007 and NK72523, Colorado College and UC-Colorado Springs during his stay at Colorado College in the Fall of 2006.

The second author was supported as the Verner Z. Reed Professor of Natural Science at Colorado College from 2007 to the present.

(C)2010 American Mathematical Society Reverts to public domain 28 years from publication 423 
In this short paper we provide a stronger but partial answer to this question by showing that for semi-hereditary rings the divisibility theory can be described by way of semi-hereditary Bezout semigroups. As a by-product, this result describes both the divisibility theory of semi-hereditary rings and also of certain sub-direct products of GCD domains, including localizations of polynomial rings in any set of variables over fields. It also provides an overview of the nature of semi-hereditary (Bezout) rings which form a proper subclass between rings with global dimension at most 1 and rings with weak dimension at most 1 . Notably, the compactness of the minimal spectrum describes semi-hereditary rings inside rings with weak dimension at most 1 . In other words, a ring of weak dimension at most 1 is semi-hereditary if and only if its minimal spectrum is compact. Note that the divisibility theory in a $G C D$ domain is a semigroup of principal ideals together with the binary operations GCD and LCM. Therefore semi-hereditary B-semigroups describe divisibility in much larger classes of rings, in particular for semi-hereditary rings.

In the first part we characterize semi-hereditary B-semigroups by their geometric structure, that is, the space of minimal m-prime filters endowed with the Zariski topology. The corresponding versions of these results (with one exception) are well-known for semiprime rings [14, [10. However, the focus of our work is on semigroups, and most of the proofs found in [14 are not direct, are mainly homological in nature, and involve localization techniques. We provide direct elementary proofs in the setting of semigroups which also hold for semiprime rings.

In the second part, the geometry of a semi-hereditary B-semigroup $S$ and its subsemigroup $S_{1}$ of non-zero-divisors is used to obtain a semi-hereditary Bezout ring $R$ with a given divisibility theory $S$. The ring $R$ is a well-determined subring of the ring of continuous functions from the minimal spectrum of $S$ into the quotient field of the semigroup algebra of $S_{1}$ endowed with the discrete topology. In the tradition of work by Arens and Kaplansky (see Chapter IX.6 [12]) on the representation of certain biregular rings, our construction provides results in the spirit of the representation of commutative $C^{*}$-algebras by Gelfand and Naimark (see Theorem 4.29 [4) as rings of continuous functions. Our work naturally suggests future efforts to characterize the semi-hereditary Bezout rings obtained by our construction and belongs to the rich theory describing the divisibility of rings. For the divisibility of various classes of rings we refer to the recent book [8] and papers [7, 9]. The question as to whether Bezout semigroups characterize the divisibility theory of Bezout rings is still open.

A word about terminology. All structures are commutative, and rings have the identity element 1. A ring is called Bezout if every finitely generated ideal is principal. The extension of a semigroup $S$ by a zero element will be denoted by $S^{\bullet}$.

\section{BASIC NOTIONS AND PRELIMINARY RESUltS}

For the benefit of the reader and for the sake of completeness we recall some basic definitions and notation and include some crucial results from [2. The development of and comments on this supporting material can be found in full detail in [2.

Definition 1.1 (see Section $4[3$ and Definition $1.1[2]$ ). A Bezout semigroup $S$ (in short a $B$-semigroup) is a commutative monoid $S$ with 0 such that the divisibility relation $a \mid b \Longleftrightarrow a S \supseteq b S$ is a partial order, called a natural partial order, inducing a distributive lattice on $S$, with a multiplication distributive on both meets and 
joins, and $S$ is hyper-normal, meaning that for any $a, b, d=a \wedge b \in S, a=d a_{1}$ there is $b_{1} \in S$ satisfying $a_{1} \wedge b_{1}=1, b=d b_{1}$.

A Bezout semigroup $S$ is semi-hereditary if for each $a \in S$ there is $e^{2}=e \in S$ with $e S=a^{\perp}=\{x \in S \mid a x=0\}$.

A B-semigroup is called cancellative if nonzero elements form a cancellative monoid.

Since divisibility is a partial order, 1 is the unique invertible element in a Bsemigroup. The most natural example of B-semigroups is the extension $P^{\bullet}$ of the positive cone $P$ of an abelian lattice-ordered group by an extra zero element. Therefore divisibilities of Bezout domains are cancellative B-semigroups. More generally, Proposition 1.4 [2] establishes

Proposition 1.1. If $R$ is a Bezout ring, then the divisibility theory $S_{R}$ of $R$ is a B-semigroup.

For the sake of completeness and due to the importance of this result, we include a direct, detailed proof which does not appear in 2 .

Proof. For any $a, b, z \in R$ and arbitrary $c \in a R \cap b R$, let $d R=a R+b R, d=$ $a x+b y, a=d a_{1}, b=d b_{2}, c=a c_{1}$ with some $a_{1}, b_{2}, c_{1}, d, x, y \in R$. Then $a b_{2}=$ $d a_{1} b_{2}=a_{1} b \in a R \cap b R$ and $b R=b R+c R+b c_{1} R=d b_{2} R+a c_{1} R+b c_{1} R=$ $d b_{2} R+c_{1}(a R+b R)=d b_{2} R+d c_{1} R=d\left(b_{2} R+c_{1} R\right)$, and hence $a_{1} b R=d a_{1}\left(b_{2} R+\right.$ $\left.c_{1} R\right) \supseteq d a_{1} c_{1} R=c R$. Therefore $a R \cap b R$ is the principal ideal $a_{1} b R$. Consequently $S_{R}$ is a sublattice of the ideal lattice of $R$ which is a distributive lattice under reverse inclusion. Thus $S_{R}$ is a distributive lattice under the natural partial order. It is trivial that ideal multiplication is distributive on meets, i.e. sums of ideals. In view of $(z R)(a R)+(z R)(b R)=z a R+z b R=z(a R+b R)=z d R$ we have $(z R)(a R) \cap(z R)(b R)=z a R \cap z b R=z a_{1} b R=(z R)\left(a_{1} b R\right)=(z R)(a R \cap b R)$, which shows that multiplication is also distributive on joins. Moreover, if $b_{1}$ is any generator of the principal ideal $\left(1-a_{1} x\right) R+b_{2} R$, then $d\left(1-a_{1} x\right)=d-a x=b y \in b R$ implies $b R=(d R)\left(b_{1} R\right), b_{1} R+a_{1} R=R$, from which hyper-normality follows.

Remark 1.2. It is important to emphasize in the above proof that the ideals generated by $b$ and $d b_{1}$ coincide although $b$ and $d b_{1}$ are not, in general, equal. That is, Bezout rings are not necessarily Hermitean rings. Moreover, ideal multiplication is obviously distributive on sums (on meets of ideals) but generally not on joins (on intersections of ideals). In fact, by Lemma 2 [13] a domain is a Prüfer domain if and only if ideal multiplication is distributive on intersections of ideals. Note that Jensen's result also holds in the more general setting of semiprime rings. Namely, a ring without nonzero nilpotent elements has a distributive ideal lattice if and only if ideal multiplication is distributive on intersections of ideals. Recall that an arithmetical ring is a ring with a distributive ideal lattice. The semiprime condition is essential. For example, the factor ring $k[x, y] /(x, y)^{2}$ of a polynomial ring in two variables over a field is obviously not an arithmetical ring, but ideal multiplication is clearly distributive over intersections of ideals. For further comments we refer to Remarks 1.4 [2].

Examples 1.2. One can obtain further classical examples of B-semigroups (see Examples 1.3 and Proposition 1.4 [2]) by considering 
(1) semigroups of principal ideals in unique factorization domains where meets (given as principal ideals generated by greatest common divisors) are not necessarily sums of ideals, e.g., in $k[x, y]$ one has $(x) \wedge(y)=(1) \neq(x, y)$;

(2) semigroups of ideals in Dedekind domains;

(3) semigroups of finitely generated ideals in Prüfer domains, or more generally, in arithmetical rings.

Corollary 1.3. The divisibility theory $S_{R}$ of a semi-hereditary ring $R$ is a semihereditary B-semigroup.

Proof. By Theorem VI.2.9 [5] a semi-hereditary ring $R$ has weak dimension at most 1, implying (in view of the main result in [13]) that $R$ is an arithmetical ring. Therefore $S_{R}$ is order-isomorphic to the divisibility theory of an appropriate Bezout ring according to Corollary 8.1 [1]. Hence $S_{R}$ is a B-semigroup by Proposition 1.1. Since the annihilator of a finitely generated ideal is a finite intersection of the annihilators of principal ideals, it is generated by an idempotent, provided that the ring is semi-hereditary. It follows that $S_{R}$ is a semi-hereditary B-semigroup.

An obvious consequence of hyper-normality is

Corollary 1.4. In a Bezout semigroup, idempotents form a Boolean algebra.

Proof. For any idempotent $e=e^{2}$ hyper-normality implies an element $f$ with $e \wedge f=1, e f=0$, whence $f^{2}=f, e \vee f=0$; i.e., $f$ is the complement of $e$, completing the proof.

When working with B-semigroups, one has to deal with filters and the so-called $m$-prime filters instead of the usual ideals and prime ideals in semigroup theory. In view of the lattice structure, m-prime filters reveal additional information via a hidden addition induced by the meet operation. For example, the multiplicative semigroup of the natural numbers has uncountably many ideals, but the ring of integers has only countably many ideals. Recall that a filter in a B-semigroup $S$ is a subset $F$ closed under $\wedge$ such that if $a \in F, b \in S$, and $a \leq b$, then $b \in F$. Therefore every filter is also an ideal of the semigroup $S$ (in the usual sense) with respect to multiplication. A filter $I$ is said to be $m$-prime if $a b \in I$ implies $a \in I$ or $b \in I$. Thus m-prime filters are particular cases of prime ideals in weak ideal systems (Aubert's x-systems), and Theorem 1.5 is related to the development in Section 4.4 of Halter-Koch's book [8]. An element $s \in S$ is a zero-divisor if $s t=0$ for some $0 \neq t \in S$. An element $a \in S$ is a non-zero-divisor if it is not a zero-divisor, i.e., if $a^{\perp}=0$.

The following result is basic in our approach.

Theorem 1.5 (see Theorem $2.13[2]$ ). For every $m$-prime filter $I$ a relation $\Phi_{I}$

$$
\forall x, y \in S: x \cong y \Longleftrightarrow \exists s \notin I: x \leq y s \& y \leq x s
$$

defines a congruence whose factor $S_{\varphi}=S / \Phi_{I}$ is a naturally totally ordered Bsemigroup, and the congruence class of 0 is $K=\{x \in S \mid \exists s \notin I: s x=0\}$.

Proof. It is routine to verify that the relation $\Phi_{I}$ is a congruence and $S \backslash I$ and $K$ are sets of elements congruent to 1 and 0 , respectively. Images in $S_{\varphi}$ of $s \in S$ are denoted by $s_{\varphi}$. Given any two elements $x, y \in I$, hyper-normality allows us to write $d=x \wedge y, x=d x_{1}, y=d y_{1}, x_{1} \wedge y_{1}=1$ for some $x_{1}, y_{1} \in S$. Therefore at least one of $x_{1}, y_{1}$ does not belong to $I$. Consequently images of $x, y$ in $S_{\varphi}$ are 
comparable; i.e., $S_{\varphi}$ is totally ordered. Moreover, $x_{\varphi} \leq y_{\varphi}$ implies $x_{\varphi}=(x \wedge y)_{\varphi}$, whence $y_{\varphi}=x_{\varphi} z_{\varphi}$ for $z \in S$ with $y=(x \wedge y) z$. If the equality $y_{\varphi}=x_{\varphi} z_{\varphi}$ holds for some $x, y, z \in S$, then there exists $s \notin I$ with $x \leq x z \leq y s$, hence $x_{\varphi} \leq y_{\varphi}$. This shows that the induced order in $S_{\varphi}$ is natural. Furthermore, if $a \in I, b \in S$ have the property that $a, a b$ have the same nonzero image in $S_{\varphi}$, then by definition there is some $s \notin I$ with $a b \leq a s$. If $b \in I$, then by putting $s \leq b \vee s=c=s c_{1} \in I$ for some $c_{1} \in S$ one has that $c, c_{1} \in I$ and $0 \neq a s=a b \vee a s=a(b \vee s)=a c=a s c_{1}$. Therefore hyper-normality implies that there is an element $x \in S$ with as $x=0, c_{1} \wedge x=1$. This shows that $x \in I$ and thus $c_{1} \notin I$ and so $b$ cannot be an element of $I$, a contradiction. It follows that $b \notin I$. This implies that $S_{\varphi}$ is hyper-normal, and it is now easy to check that $S_{\varphi}$ is a B-semigroup.

Remark 1.6. For an m-prime filter $I$ the factor of $S$ by the congruence

$$
\forall x, y \in S: x \cong y \Longleftrightarrow \exists s \notin I: x \leq y s \& y \leq x s
$$

is called the localization of $S$ at $I$ (sending $S \backslash I$ to 1 ).

The nil radical of a B-semigroup $S$ is the (set-theoretical) intersection of all m-prime filters. It is routine to see that the nil radical of $S$ is just the set of all nilpotent elements. $S$ is called semiprime or reduced if its nil radical is 0 . An m-prime filter $I$ of $S$ is called minimal if it is minimal in the set of all m-prime filters of $S$ with respect to the set-theoretical inclusion. Throughout this paper $\mathcal{X}$ will denote the set of all minimal m-prime filters of $S$. It is obvious that the nil radical is also the intersection of all minimal m-prime filters.

Definition 1.3. The minimal spectrum of $S$ is the set $\mathcal{X}$ of all minimal m-prime filters endowed with the Zariski topology by taking $\left\{D_{s}=\{I \in \mathcal{X} \mid s \notin I\}, s \in S\right\}$ as a basis of open sets.

It is routine to check that $\left\{D_{s}=\{I \in \mathcal{X} \mid s \notin I\}, s \in S\right\}$ satisfies the axioms for a basis of open sets in a topological space. For the sake of completeness we provide the proofs of the following obvious but important assertions.

Proposition 1.7. Let $S$ be a reduced B-semigroup.

(1) [14, Proposition $1.1(3)] \bigcup_{I \in \mathcal{X}} I$ is the set of all zero-divisors of $R$.

(2) [14, Proposition 1.2 (1)] An m-prime filter $I$ is minimal iff for all $s \in$ $I, s^{\perp} \nsubseteq I$.

Proof. Let $I$ be a minimal m-prime filter and let $0 \neq s \in I$. By Theorem 1.5 the localization $S_{I}$ is a naturally totally ordered B-semigroup; hence it must be a Boolean algebra of two elements 1 and 0 because $S$ is reduced and $I$ is a minimal m-prime filter. This implies that there is an element $t \notin I$ with $t s=0 . t \notin I$ implies $t \neq 0$. Thus $\bigcup_{I \in \mathcal{X}} I$ consists entirely of zero-divisors. On the other hand, if $0 \neq s$ is a zero-divisor, then there exists $0 \neq t$ with $t s=0$. Since $S$ is reduced, there is a minimal m-prime filter $I$ with $t \notin I$. Therefore $s \in I$ holds because $t s=0 \in I$. This finishes the verification of (1).

If $I$ is a minimal m-prime filter and $0 \neq s \in I$, then we have already seen that there is some $t \notin I$ with $t s=0$, i.e, $s^{\perp} \neq 0$ and $s^{\perp} \nsubseteq I$. Conversely, let $I$ be an m-prime filter such that $s^{\perp} \nsubseteq I$ holds for all $s \in I$. Assume indirectly that there is another m-prime filter $J$ properly contained in $I$. Then for $s \in I \backslash J$ we have $s^{\perp} \subseteq J \subseteq I$, a contradiction. Thus, statement (2) holds. 
Remark 1.8. Although $\bigcup_{I \in \mathcal{X}} I$ is the set of all zero-divisors of $R$, there exist in general m-prime filters consisting of zero-divisors which are not minimal.

\section{Semi-hereditary B-Semigroups}

For every element $a \in S$ let $S_{a}=\left\{s \in S \mid s^{\perp}=a^{\perp}\right\}$. The following simple but basic result is proved in [2]. For the sake of clarity and because of its importance for our development, we include the proof.

Proposition 2.1 (cf. Theorem 3.5 [2]). If $S$ is a semiprime B-semigroup, then for every element $a \in S$ the subsemigroup $S_{a}$ is a cancellative semigroup closed under $\wedge$ and $\vee$. The extension $S_{a}^{\bullet}$ of $S_{a}$ by a new extra zero element 0 is a B-semigroup if and only if its induced order is natural, i.e., if $S_{a} \ni b \leq c \in S_{a}$ implies $c=b u$ for some $u \in S_{a}$.

Proof. Assume $x, y \in S_{a}$. Then $(x \wedge y)^{\perp}=x^{\perp} \cap y^{\perp}=a^{\perp}$. If $t(x \vee y)=0$, then $t x y=0$. Hence $t x \in y^{\perp}=x^{\perp}$; thus $(t x)^{2}=0 \Longrightarrow t x=0$, i.e., $t \in x^{\perp}$. Thus $S_{a}$ is a subsemigroup closed under both $\wedge$ and $\vee$. If now $x z=y z$ with $x, y, z \in S_{a}$, then $(x \wedge y) z=(x \vee y) z$ holds. Hence one can assume without loss of generality that $x<y=d x$ and it follows that $z x=d z x$. Thus $x \neq y$ would imply $d \neq 1$. If there is some $c \in x^{\perp}=a^{\perp}$ with $c \wedge d=1$, then we have $x=x \cdot 1=x(c \wedge d)=x d=y$, a contradiction. Thus there is an m-prime filter $I$ such that in the 0 -cancellative naturally totally ordered factor $S / \Phi_{I}$ we have $d_{\varphi} \neq 1,(x z)_{\varphi} \neq 0$ and the equality $(x z)_{\varphi}=d_{\varphi}(x z)_{\varphi}$, a contradiction.

Assume now that $S_{a}^{\bullet}$ is a B-semigroup and $b, c \in S_{a}, b \leq c$. Then $S_{a}$ has an identity; i.e., there is an idempotent $e \in S_{a}$ and we have $c=b d$ for some $d \in S$. This implies $c=b d=b e d=b u$ with $u=d e \in S_{a}$, as is easy to check. Conversely, $a \leq a$ implies that $a=a e$ for some $e \in S_{a}$, and hence $e=e^{2}$ is the identity of $S_{a}$. Consequently $S_{a}$, endowed with 0 , is a B-semigroup.

Corollary 2.2. Let $S$ be a B-semigroup.

(1) If for every $a \in S, S_{a}$ contains an idempotent, then $S$ is semiprime.

(2) $S$ is semi-hereditary iff $S_{a}$ contains an idempotent for each $a \in S$. In particular, semi-hereditary B-semigroups are disjoint unions of positive cones of lattice-ordered groups, whence reduced.

Proof. If $S_{a}$ contains an idempotent $e$, then $a^{\perp}=e^{\perp}=f S$ where $f$ is the orthogonal complement of $e$. This shows the equivalence claimed in assertion (2) and hence claim (1).

Remarks 2.3. (1) If $R$ is a semi-hereditary Bezout ring, then it is well-known (see 13) as a consequence of a long homological argument that $R$ is semiprime. Our proof provides a short, direct elementary justification. Note that semi-hereditary associative rings are not necessarily semiprime. For instance, upper triangular matrix rings over fields are hereditary and have nonzero nilpotent radicals.

(2) It is well-known that non-trivial lattice-ordered abelian groups are torsionfree. However, in our treatment of semi-hereditary B-semigroups, the trivial group consisting only of the identity element is lattice-ordered in the obvious way. The reason for this convention is the case of Boolean algebras which are obviously B-semigroups $S$ where $S_{a}$ is the trivial group for any element $a \in S$. 
Now we turn to the geometric structure of semi-hereditary B-semigroups. With the exception of claim (2), the ring-theoretical statements corresponding to assertions in the following theorem are well-known even for the larger class of reduced rings (see Section 1 in [14]).

Theorem 2.4. For a B-semigroup $S$ the following statements are equivalent:

(1) $S$ is semi-hereditary.

(2) $S$ is reduced and for each element $a \in S$ there exists $b \in S$ such that $a^{\perp}=b S$.

(3) $S$ is reduced and for each $a \in S$ there exists $b \in a^{\perp}$ such that $a \wedge b$ is $a$ non-zero-divisor.

(4) The minimal spectrum $\mathcal{X}$ of $S$ is compact with respect to the Zariski topology.

Remarks 2.5. (1) The condition in claim (2) that $a^{\perp}$ is a cyclic filter for each $a \in S$ means precisely that every principal filter is finitely presented. Bezout rings with this condition are exactly coherent Bezout rings.

(2) Any $e^{2}=e \in S$ together with its complement $f$ induces a direct decomposition

$$
S_{e} \times S_{f} \longrightarrow S_{1}:\left(S_{e} \ni s, t \in S_{f}\right) \longmapsto s \wedge t \in S_{1} \ni u \longmapsto(e u, f u) \in S_{e} \times S_{f} .
$$

Consequently, the structure of a semi-hereditary B-semigroup $S$ is completely described by its subsemigroup $S_{1}$, which is a positive cone of a lattice-ordered abelian group $G$, and a Boolean algebra of idempotents in $S$ such that each idempotent of $S$ induces a direct decomposition of $G$ with obvious compatibility conditions. Thus one might think that this Boolean algebra can be realized as a Boolean algebra of commuting idempotents in the endomorphism ring of the abelian group $G$. However, this is not necessarily the case in view of Remark 2.3 (2). Here is another simple example to the contrary. Let $R$ be the subring in the direct product $\prod_{i \in \mathbb{N}} \mathbb{Q}$ of countably infinitely many copies of the field $\mathbb{Q}$ of rationals generated by their direct sum; i.e., $R$ is a subring of sequences, all but finitely many of whose entries are equal to an integer. Such a ring $R$ is a semi-hereditary Bezout ring. Let $S$ be a semigroup of divisibility of $R$. Then one can verify that a Boolean algebra of idempotents of $S$ is isomorphic to a Boolean algebra generated by finite subsets of $\mathbb{N}$ with respect to unions and intersections and $S_{1}$ is just the multiplicative semigroup $\mathbb{N}$. However, every idempotent $e$ of $S$ induces the same identity map of $\mathbb{N}$, and $S_{e}$ consists either of one element or is isomorphic to $S_{1}$.

Proof. The implication $1 \Longrightarrow 2$ is trivial by Corollary 2.2 (2) and the definition of semiheredity.

The implication $2 \Longrightarrow 3$ is obvious.

$3 \Longrightarrow 4$. To show the compactness of $\mathcal{X}$, it is enough to see that every open covering of $\mathcal{X}$ consisting of open sets of the form $D_{a_{i}}$ contains a finite subcollection that also covers $\mathcal{X}$ because $\left\{D_{s} \mid s \in S\right\}$ forms a basis of open sets for the Zariski topology. Let $F$ be the filter generated by all of the $a_{i}$. One can assume that all the $a_{i}$ are zero-divisors because $D_{a}=\mathcal{X}$ holds for a non-zero-divisor $a \in S$. If $F$ does not contain non-zero-divisors, then $F$ can be extended to an m-prime filter $I$ disjoint from $S_{1}$. Then $I$ must be a minimal m-prime filter by Proposition $1.7(2)$. 
In fact, for any element $s \in I$ there is $t \in s^{\perp}$ with the non-zero-divisor $s \wedge t$, hence $t \notin I$. Consequently $s^{\perp} \nsubseteq I$ holds. Therefore we have shown that $I \in \mathcal{X}$. However, $I \notin \bigcup D_{a_{i}}$ holds because all the $a_{i}$ belong to $I$; i.e., $\left\{D_{a_{i}}\right\}$ is not an open covering of $\mathcal{X}$, a contradiction. Henceforth $F$ must contain a non-zero-divisor $d$; consequently, there are finitely many $a_{i}$ 's, say $a_{1}, \cdots, a_{n}$, such that $d$ is contained in the filter generated by $a_{1}, \cdots, a_{n}$. This shows $\bigcup_{i=1}^{n} D_{a_{i}}=\mathcal{X}$, which completes the verification that $\mathcal{X}$ is compact.

$4 \Longrightarrow 1$. For an arbitrary element $a \in S$ and $I \in \mathcal{X}$ the equality $a a^{\perp}=0 \in I$ implies either $a \in I$ or $a \notin I$. The latter case implies $I \in D_{a}$. The first case shows that there is one $b \in a^{\perp}$ with $b \notin I$ in view of Proposition 1.7(2), thus $I \in D_{b}$. Therefore $\left\{D_{a}, D_{s} \mid s \in a^{\perp}\right\}$ is an open covering of $\mathcal{X}$. By the compactness of $\mathcal{X}$ there are finitely many $s \in a^{\perp}$, and so, by taking their meet $b \in a^{\perp}$, we have $D_{a} \cup D_{b}=\mathcal{X}$. Consequently $d=a \wedge b$ is a non-zero-divisor. By the hyper-normality condition there are $a_{1}, b_{1} \in S$ such that $a_{1} \wedge b_{1}=1, a=d a_{1}, b=d b_{1}$. On the other hand, $0=a b=d^{2} a_{1} b_{1}$ implies $a_{1} b_{1}=0$ because $d$ is a non-zero-divisor. Therefore $a_{1}^{2}=a_{1}, b_{1}^{2}=b_{1}$ and $b_{1} S=a^{\perp}$ hold. This completes the proof of the theorem.

From the proof of the implication $3 \Longrightarrow 4$ one obtains the following assertion.

Corollary 2.6. If $F$ is a filter consisting of zero-divisors in a semi-hereditary Bsemigroup, then $F$ is contained in a minimal m-prime filter. In particular, minimal m-prime filters in semi-hereditary B-semigroups are maximal m-prime filters consisting of zero-divisors. Therefore, a reduced B-semigroup is semi-hereditary if and only if the set of minimal m-prime filters coincides with the set of maximal m-prime filters of zero-divisors.

Remark 2.7. If a minimal m-prime filter $F$ in a B-semigroup $S$ is at the same time a maximal m-prime filter, then for each $a \notin F$ there is obviously $b \in F$ such that $a \wedge b$ is a non-zero-divisor but $b$ is, in general, not annihilated by $a$.

It is well-known (see [13]) that a ring has weak dimension at most 1 if and only if its localization at each prime ideal is a valuation domain. The following result provides the topological property of the minimal spectrum that characterizes semi-hereditary rings among commutative rings with weak dimension at most 1 .

Corollary 2.8. A commutative ring $R$ has weak dimension at most 1 and its minimal spectrum is compact if and only if $R$ is semi-hereditary.

Proof. By Corollary 1.3 and Theorem 2.4 it is enough to prove sufficiency. Let $S$ be the divisibility of $R$. Then it is obvious that the minimal spectra of $S$ and $R$ are canonically homeomorphic. Therefore, again by Theorem 2.4, $S$ is a semi-hereditary B-semigroup. This implies that the annihilator $I^{\perp}$ of any finitely generated ideal of $R$ is also a finitely generated idempotent ideal. Consequently, $I^{\perp}$ is generated by an idempotent. In particular, every cyclic ideal of $R$ is projective. Moreover, any finitely generated ideal $I$ is flat because its localization $I_{P}$ at any prime ideal $P$ is either a free module of rank 1 over a valuation domain $R_{P}$ or 0 in view of the equality $I^{\perp}=e R$ for some $e^{2}=e \in R$. Therefore $R$ is semi-hereditary by Proposition 2.7 [14].

Proposition 2.9. For every open and closed subset $U$ in the minimal spectrum $\mathcal{X}$ of a semi-hereditary B-semigroup there is precisely one idempotent $e^{2}=e$ with $U=D_{e}$. 
Proof. Since $U$ is open, $U=\bigcup D_{s_{i}}$ for appropriate elements $s_{i}$. Therefore $\{X \backslash$ $\left.U, D_{s_{i}}\right\}$ is an open covering of $X$ because $U$ is closed. Hence the compactness of $X$ shows that there are finitely many $s_{i}$ 's, say $s_{1}, \cdots, s_{n}$, such that $U=\bigcup_{i=1}^{n} D_{s_{i}}$ which implies $U=D_{s}$ if $s=\wedge_{i=1}^{n} s_{i}$. By assumption there is an idempotent $e$ with $s^{\perp}=e^{\perp}$. The inclusion $D_{s} \subseteq D_{e}$ obviously holds because $e \leq s$. If $I \in D_{e} \subseteq \mathcal{X}$, i.e., $e \notin I$ and $s \in I$, then for the orthogonal complement $f^{2}=f$ of $e$ the element $s \wedge f \in I$ is a non-zero-divisor, a contradiction by Proposition 1.7 (2). Thus $s \notin I$, which shows $D_{e} \subseteq D_{s}$. Hence $D_{e}=D_{s}$. If $D_{e}=D_{g}$ holds for some other idempotent $g^{2}=g$, then $D_{e}=D_{g}=D_{e \wedge g}=D_{e \vee g}$ and hence $e=e \wedge g=e \vee g=g$, which completes the proof.

Theorem 2.10. The minimal spectrum $\mathcal{X}$ of a semi-hereditary B-semigroup $S$ is a Stone space, i.e., a totally disconnected compact Hausdorff space. If $B$ is the Boolean algebra of idempotents in $S$, then the map $I \in \mathcal{X} \mapsto I \cap B$ is a homeomorphism between $\mathcal{X}$ and the prime spectrum $\mathcal{M}$ of $B$.

Proof. Let $\mathcal{X} \ni I \neq J \in \mathcal{X}$. Then there is an element $a \in I \backslash J$. By assumption there is an idempotent $e \in S$ such that $a^{\perp}=e^{\perp}$. If $f^{2}=f$ is the orthogonal complement of $e$, then $I \in D_{f}$ and $J \in D_{e}$ imply that $\mathcal{X}$ is Hausdorff and totally disconnected. It is clear that $I \cap B$ is a prime ideal of the Boolean algebra $B$; i.e., it is easy to check that the map $I \in \mathcal{X} \mapsto J=I \cap B \in \mathcal{M}$ is well-defined with inverse the map which carries $J \in \mathcal{M}$ to $\bigcup_{e \in J} S_{e} \in \mathcal{X}$. The last assertion then follows immediately from the definitions of the topologies of $\mathcal{X}$ and $\mathcal{M}$.

\section{THE REPRESENTATION THEOREM}

The main aim of this section is to prove the following theorem.

Theorem 3.1. A semigroup $S$ is the divisibility theory of a semi-hereditary Bezout ring if and only if it is a semi-hereditary B-semigroup.

Proof. The necessity that the divisibility theory of a semi-hereditary Bezout ring is a semi-hereditary B-semigroup is obvious by Proposition 1.1. For the converse, we will construct a semi-hereditary Bezout ring with an arbitrary pre-described semi-hereditary B-semigroup $S$. For this aim, let $L$ be an arbitrary field. It is well-known (see Theorem 4.5.1 [6]) that the localisation $A$ of the semigroup algebra $L S_{1}$ at the set

$$
\left\{\sum_{i=1}^{i=n} k_{i} s_{i} \mid 0 \neq k_{i} \in L, s_{i} \in S_{1}, \bigwedge_{i=1}^{n} s_{i}=1, n \in \mathbb{N}\right\}
$$

of primitive elements is a Bezout domain whose divisibility semigroup is just $S_{1}$ extended with the new extra zero element 0 . Moreover, $S_{1}$ is identified as a subsemigroup of $A$ under multiplication. Furthermore, let $K$ be the quotient field of $A$. For every $e^{2}=e \in S$ let $f^{2}=f \in S$ be its orthogonal complement. Then $(f S)_{1}$ is order isomorphic to $\left\{e \wedge t \mid t \in S_{f}=\left(e^{\perp}\right)_{1} \wedge e\right\}$, which is a multiplicatively closed subset of $S_{1} \subseteq A$. Let $A_{e}$ be the localisation of $A$ at this subset. Thus we have the equality $A_{1}=A$, and moreover $A_{e}$ is a subring of $A_{g}$ for every idempotent $g \in S$ satisfying $g e=g$. We further claim 
Lemma 3.2. The divisibility semigroup of $A_{e}$ is isomorphic to $S_{e}^{\bullet}$ under the map $f \wedge s \mapsto s \in S_{e}$.

Proof. For any $t \in\left(e^{\perp}\right)_{1}=(S f)_{1}=S_{f}$ and $s \in S_{e}$ in $A$, the invertibility of $t \wedge e \in A_{e}$ and the equalities

$$
(t \wedge e)(f \wedge s)=(t f \wedge t s) \wedge(e f \wedge e s)=(t \wedge 0) \wedge(0 \wedge s)=t \wedge s
$$

imply that $(t \wedge s) A_{e}=(f \wedge s) A_{e}$, from which the assertion follows.

We are now in position to construct a semi-hereditary Bezout ring $R$ whose semigroup of divisibility is $S$. Let $C_{K}(\mathcal{X})$ denote the ring of all continuous functions from the minimal spectrum $\mathcal{X}$ of $S$ into the field $K$ endowed with the discrete topology. If $U$ is an open and closed subset of $\mathcal{X}$, then a characteristic function $\chi_{U}$ which is 1 on $U$ and 0 on $\mathcal{X} \backslash U$ is trivially an element of $C_{K}(\mathcal{X})$. Since $\mathcal{X}$ is compact, Hausdorff and totally disconnected, a function from $\mathcal{X}$ to $K$ is continuous if it is a step function associated to a partition of $\mathcal{X}$ as a finite disjoint union of open subsets, i.e., a linear combination $\sum_{i=0}^{i=n-1} a_{i} \chi_{U_{i}}$ of characteristic functions $\chi_{U_{i}}$ with respect to some finite partition $\mathcal{X}=\bigcup_{i=0}^{n-1} U_{i}, n \in \mathbb{N}$, of $\mathcal{X}$ into pairwise disjoint open (and hence also closed) subsets $U_{i}$ where the $\left\{a_{i} \in K\right\}$ are $n$ distinct elements of $K$. The partition is finite because the topology on $K$ is discrete. On the other hand, every open and closed subset $U$ of $\mathcal{X}$ is of the form $D_{e}$ for a unique idempotent $e^{2}=e \in S$. Therefore, for the sake of simplicity, the characteristic function $\chi_{D_{e}}$ will be denoted as $\chi_{e}$. Hence a function $\varphi: \mathcal{X} \longrightarrow K$ is continuous if and only if there are finitely many pairwise orthogonal idempotents $e_{i}$ with $\wedge_{i=0}^{n-1} e_{i}=1 \mathrm{such}$ that $\varphi$ is equal to the linear combination $\sum_{i=0}^{i=n-1} a_{i} \chi_{e_{i}}$. For the sake of convenience we always set $a_{0}=0$; i.e., $\bigcup_{i=1}^{n-1} D_{e_{i}}=(\varphi)^{-1}(K \backslash\{0\})$ is the support of $\varphi$ and $D_{e_{0}}$ denotes the subset $(\varphi)^{-1}(0) \subseteq \mathcal{X}$. Note that the subset $D_{e_{0}}$ can be the empty set. We define $R$ to be a subset of $C_{K}(\mathcal{X})$ consisting of linear combinations $\sum_{i=0}^{i=n-1} a_{i} \chi_{e_{i}}$ satisfying $\wedge_{i=0}^{n-1} e_{i}=1$ where $e_{i}$ are pairwise orthogonal idempotents and $a_{i} \in A_{e_{i}}$ for every index $i$.

Lemma 3.3. $R$ is a subring of $C_{K}(\mathcal{X})$.

Proof. It is clear that $R$ contains the identity element $\chi_{\mathcal{X}}$, its scalar multiples by elements of $A$, and the zero element $\chi_{\emptyset}$ of $C_{K} \mathcal{X}$. Therefore we have to show only that $R$ is closed under addition and multiplication; i.e., $\varphi, \theta \in R$ imply $\varphi+\theta, \varphi \theta \in R$. Write $\varphi=\sum_{i=0}^{i=n-1} a_{i} \chi_{e_{i}}$ and $\theta=\sum_{j=0}^{i=m-1} b_{j} \chi_{f_{j}}$ as linear combinations of characteristic functions such that $a_{0}=0=b_{0}, a_{i} \in A_{e_{i}}, b_{j} \in A_{f_{j}}$, and $e_{i}$ as well as $f_{j}$ are pairwise orthogonal idempotents of $S$ with $\wedge_{i=0}^{n-1} e_{i}=1=\wedge_{j=0}^{m-1} f_{j}$, respectively. Note that some of the subsets $D_{e_{0}}, D_{f_{0}}$ can be empty. It is obvious that $\left\{D_{e_{i}} \cap D_{f_{j}}=\right.$ $\left.D_{e_{i} \vee f_{j}}=D_{e_{i} f_{j}}\right\}$ is a partition of $\mathcal{X}$ induced by pairwise orthogonal idempotents $e_{i} \vee f_{j}=e_{i} f_{j}$. It follows that $R$ is closed under multiplication and addition by noting that $A_{e_{i}}, A_{f_{j}}$ are subrings of $A_{e_{i} f_{j}}$, which completes the proof. 
We are now in position to show that $R$ is a semi-hereditary Bezout ring whose semigroup of divisibility is $S$. Consider an arbitrary element $\varphi=\sum_{i=0}^{i=n-1} a_{i} \chi_{e_{i}} \in R$ where $a_{0}=0, a_{i} \in A_{e_{i}}$, and $e_{i}$ are pairwise orthogonal idempotents of $S$ with $\wedge_{i=0}^{n-1} e_{i}=1$. Note that $(\varphi)^{-1}(0)=D_{e_{0}}$ can be empty. Let $e=\wedge_{i=1}^{n-1} e_{i}$. Since $A_{e_{i}}$ is a Bezout domain whose semigroup of divisibility is $S_{e_{i}}^{\bullet}$, there is $s_{i} \in S_{e_{i}}$ such that $a_{i} A_{e_{i}}=\left(f_{i} \wedge s_{i}\right) A_{e_{i}}$ where $f_{i}$ is the orthogonal complement of $e_{i}$ in $S$. Let $s=\wedge_{i=1}^{n-1} s_{i}$. The equalities

$$
\left(s \wedge e_{0}\right) \chi_{e}=\sum_{i=1}^{i=n-1}\left(s \wedge e_{0}\right) \chi_{e_{i}},\left(s \wedge e_{0}\right) \chi_{e_{i}} A_{e_{i}}=\left(f_{i} \wedge s_{i}\right) A_{e_{i}}=a_{i} A_{e_{i}}
$$

imply $\varphi R=\left(s \wedge e_{0}\right) \chi_{e} R$. Therefore to finish the proof of the theorem, it is enough to see that $R$ is a Bezout ring, i.e, to show that every ideal generated by two elements $\varphi, \theta$ is principal. As in the proof of Lemma 3.3 write $\varphi=\sum_{i=0}^{i=n-1} a_{i} \chi_{e_{i}}$ and $\theta=\sum_{j=0}^{i=m-1} b_{j} \chi_{f_{j}}$ as linear combinations of characteristic functions such that $a_{0}=0=b_{0}, a_{i} \in A_{e_{i}}, b_{j} \in A_{f_{j}}$, where $e_{i}$ and $f_{j}$ are sets of pairwise orthogonal idempotents of $S$ with $\wedge_{i=0}^{n-1} e_{i}=1=\wedge_{j=0}^{m-1} f_{j}$, respectively. Note that some of the subsets $D_{e_{0}}, D_{f_{0}}$ can be empty. It is obvious that $\left\{D_{e_{i}} \cap D_{f_{j}}=D_{e_{i} \vee f_{j}}=D_{e_{i} f_{j}}\right\}$ is a partition of $\mathcal{X}$ induced by pairwise orthogonal idempotents $e_{i} \vee f_{j}=e_{i} f_{j}$. In each ring, the ideal $A_{e_{i} f_{j}}$ generated by $a_{i}$ and $b_{j}$ is principal. This implies that $\varphi R+\theta R$ is a principal ideal, and the proof of Theorem 3.1 is complete.

By Proposition 1.3 the divisibility theory of semi-hereditary rings is described in the following corollary.

Corollary 3.4. The divisibility theory of a semi-hereditary ring is a semi-hereditary Bezout semigroup, and any semi-hereditary Bezout semigroup can be realized as the divisibility theory of a semi-hereditary Bezout ring.

\section{ACKNOWLEDGMENT}

The authors are grateful to the referee for calling their attention to Halter-Koch's work on the divisibility theory of rings.

\section{REFERENCES}

1. D. D. Anderson, Multiplication ideals, multiplication rings, and the ring $R(X)$, Canad. J. Math. 28 (1976), 760 - 768. MR0424794(54:12752)

2. P. N. Ánh, L. Márki, P. Vámos, Divisibility theory in commutative rings: Bezout semigroups, manuscript.

3. B. Bosbach, Representable divisibility semigroups, Proc. Edinburgh Math. Soc. 34 (1991), 45-64. MR 1093175 (92m:20056)

4. R. G. Douglas, Banach algebra techniques in operator theory, GTM 179, $2^{\text {nd }}$ ed., SpringerVerlag, New York, 1998. MR 1634900 (99c:47001)

5. H. Cartan and S. Eilenberg, Homological algebra, Princeton, 1956. MR0077480 (17:1040e)

6. A. M. W. Glass and W. Charles Holland (eds), Lattice-ordered groups: advances and techniques, Kluwer Academic Publishers, 1989. MR.1036072 (91i:06017)

7. F. Halter-Koch, A characterization of Krull rings with zero divisors, Archivum Math. 29 (1993), 119 - 122. MR 1242634(94h:13012)

8. F. Halter-Koch, Ideal systems. An introduction to multiplicative ideal theory, Marcel Dekker, 1998. MR $1828371(2001 \mathrm{~m}: 13005)$ 
9. F. Halter-Koch, Construction of ideal systems with nice Noetherian properties, in NonNoetherian commutative ring theory (eds. S. T. Chapman and S. Glaz), pp. $271-285$, Kluwer, 2000. MR $1858166(2002 \mathrm{~h}: 13002)$

10. M. Henriksen and M. Jerison, The space of minimal prime ideals of a commutative ring, Trans. Amer. Math. Soc. 115 (1965), 110 - 130. MR0194880 (33:3086)

11. N. Jacobson, Structure of rings, revised edition, AMS, 1964. MR0222106 (36:5158)

12. P. Jaffard, Les système d'idéaux, Dunod, Paris, 1960. MR0114810 (22:5628)

13. C. U. Jensen, A remark on arithmetical rings, Proc. Amer. Math. Soc. 15 (1964), 951-953. MR0179197 (31:3446)

14. E. Matlis, The minimal prime spectrum of a reduced ring, Ill. J. Math. 27 (1983), 353-391. MR698302 (84h:13010)

15. M. H. Stone, Applications of the theory of Boolean rings to general topology, Trans. Amer. Math. Soc. 41 (1937), 375-481. MR1501905

Rényi Institute of Mathematics, Hungarian Academy of Sciences, 1364 Budapest, Pf. 127, Hungary

E-mail address: anh@renyi.hu

Department of Mathematics and Computer Science, Colorado College, Colorado Springs, Colorado 80903

E-mail address: msiddoway@coloradocollege.edu 